\title{
DUST VS. COLOR GRADIENTS IN ELLIPTICAL GALAXIES
}

\author{
PAUL GOUDFROOIJ \\ European Southern Observatory, Karl-Schwarzschild-Strasse 2, \\ D-85748 Garching bei München, Germany
}

Abstract. Color gradients in elliptical galaxies are commonly interpreted as being due to stellar population gradients (e.g., Davies et al. 1993, MNRAS 262,650 ). Here, I show that elliptical galaxies should generally contain a diffusely distributed component of dust, in addition to the "visible" component which is in the form of dust lanes or patches (see Fig. 1a). Employing a multiple scattering model for the dust, the presence of this newly postulated dust component is found to imply significant radial color gradients (see Fig. 1b). This should be taken seriously in the interpretation of color gradients in elliptical galaxies. This poster paper is based upon a (much!) more elaborate article by Goudfrooij \& de Jong (1995, A\&A 298, 784).
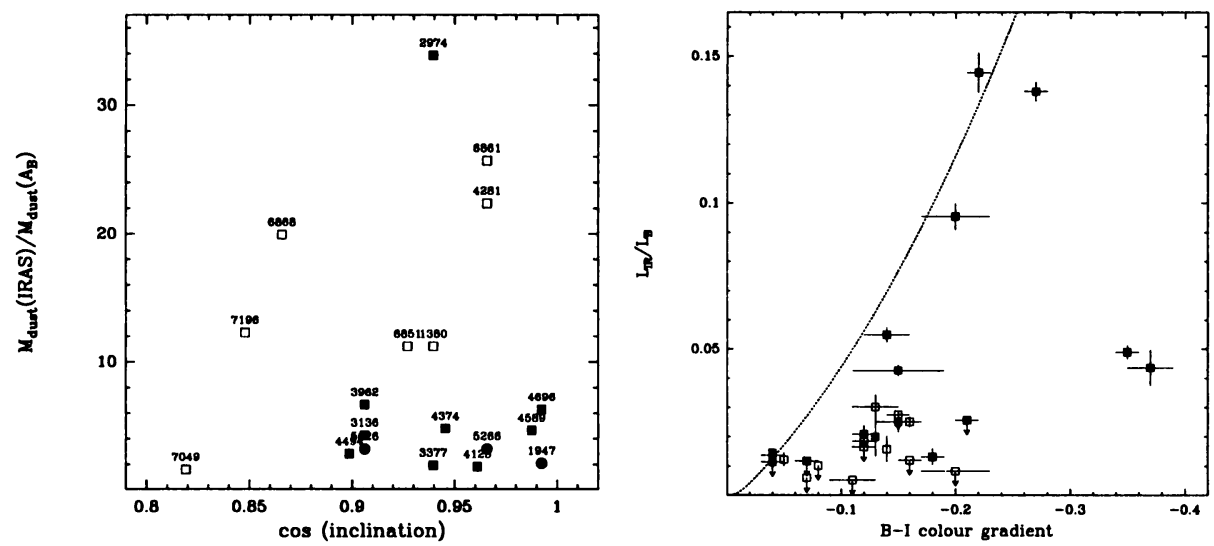

Figure 1. (A, left) The quotient of the dust mass derived from the IRAS data and the dust mass derived from optical extinction versus the cosine of the inclination angle of the dust lane in elliptical galaxies containing regular dust lanes (see Goudfrooij \& de Jong 1995). The NGC designations of the galaxies involved are indicated. (B, right) The relation of $L_{I R} / L_{B}$ with $\Delta(B-I) / \Delta(\log \mathrm{r})$ for elliptical galaxies in the "RSA sample" (cf. Goudfrooij et al. (1994, A\&AS 104, 179)). Filled squares represent galaxies showing optical evidence for dust, and open squares represent galaxies without optical evidence for dust. Arrows indicate upper limits to $L_{I R} / L_{B}$. The dotted line represents the color gradient expected from differential extinction by a diffuse distribution of dust (see text). 